\section{The Glass Transition of Trinitrotoluene (TNT) by Flash DSC}

\author{
Nabila Shamim, Yung P.Koh, Sindee L. Simon and Gregory B. McKenna* \\ Department of Chemical Engineering \\ Texas Tech University \\ Lubbock, TX 79409-3121 USA
}

\{October 3, 2015\}

\title{
ABSTRACT
}

Flash differential scanning calorimetry has been used to determine the glass transition response of the rapidly crystallizing energetic material, TNT. After heating at $600 \mathrm{~K} / \mathrm{s}$, it was found that for cooling rates from $1000 \mathrm{~K} / \mathrm{s}$ to $10 \mathrm{~K} / \mathrm{s}$ the glass transition changes from $247.5 \mathrm{~K}$ to $239.3 \mathrm{~K}$. The dynamic fragility index was determined to be $\mathrm{m}=62 \pm 6$ and the activation energy determined from the range of cooling rates in which vitrification occurred was found to be $290 \pm 16 \mathrm{~kJ} / \mathrm{mol}$. Crystallization was found to occur during cooling at rates below $0.3 \mathrm{~K} / \mathrm{s}$, whereas cold crystallization was found on subsequent heating after cooling between $10 \mathrm{~K} / \mathrm{s}$ and $30 \mathrm{~K} / \mathrm{s}$. At cooling rates of $100 \mathrm{~K} / \mathrm{s}$ and above, and for the same heating rate of $600 \mathrm{~K} / \mathrm{s}$ investigated, the glass transition event was observed, but cold crystallization during heating did not occur. Hence, the crystallization behavior of the fully amorphous TNT upon heating depends on the cooling rate and vitrification path.

Keywords: TNT; glass transition; chip calorimetry; flash DSC; cold crystallization

${ }^{*}$ Corresponding author. e-mail: greg.mckenna@ttu.edu 


\section{INTRODUCTION}

There is significant interest in creating novel materials through physical, rather than chemical, methods. In this respect, taking a rapidly crystallizing material that is generally used in the crystalline state, one may find advantages if the material can be made vitreous by avoiding crystallization. To this end, we have begun a series of works to attempt to make energetic materials that are vitreous in nature with the intent that the defect nature of the glassy material may provide improved properties, such as improved handling and stability or enhanced combustion properties.

In the present work, we use a baseline energetic material, trinitrotoluene (TNT) to demonstrate that such a material can be made vitreous, i.e., can be made into a glass. The method chosen for the demonstration is to use flash differential scanning calorimetry (DSC) to provide a means of cooling the material at a sufficiently high rate that vitrification will occur rather than crystallization. As shown subsequently, the rate at which TNT can be cooled to form a glass is outside the range of cooling rates achievable by normal DSC, but not so high that the flash-type of chip calorimeter is pushed to its limits, i.e., the required cooling rate is nearer to $10 \mathrm{~K} / \mathrm{s}$ rather than $10^{4} \mathrm{~K} / \mathrm{s}$ that can be achieved with such a calorimeter. In addition to the first direct observation and reporting of the glass transition temperature behavior of TNT, we also find that the crystallization behavior of the TNT from the glassy state is different depending on the cooling rate used to make the glass. While this is a finding that has been seen in, e.g., metallic glass-formers, its observation in the mono-component TNT suggests that fully understanding such phenomena will provide a potential path to control material behavior by varying the route to vitrification.

\section{EXPERIMENTAL METHODS}

\section{Materials}

The TNT used in this project was provided by Austin Explosives Company and was used in the present experiment after purification by crystallization from acetone. Silver paste from Ted Pella, Inc. (PELCO High Performance Silver Paste, Product number 16047) was used to create a "reservoir" for the TNT on the chip calorimeter to hold the TNT in place when it was in the liquid state.

\section{Calorimetry}

The calorimetric measurements were made using a Mettler Toledo Flash DSC 1 with Freon intercooler and nitrogen purge. Both heating and cooling scans were made over a temperature range from 178.2 $\mathrm{K}$ to $372.2 \mathrm{~K}\left(-95^{\circ} \mathrm{C}\right.$ to $\left.99^{\circ} \mathrm{C}\right)$. The cooling rates varied from $0.1 \mathrm{~K} / \mathrm{s}$ to $1000 \mathrm{~K} / \mathrm{s}$ while the heating rate was kept constant at $600 \mathrm{~K} / \mathrm{s}$. (For comparison, conventional DSC is commonly used at ranges of heating or cooling rates from 0.01 to $30 \mathrm{~K} / \mathrm{min}$, i.e., $1.7 \times 10^{-4}$ to $0.5 \mathrm{~K} / \mathrm{s}$. [1]). Calibration of the 
instrument was performed following the manufacturer procedures and using indium as described by Gao,

62 et al. [2].

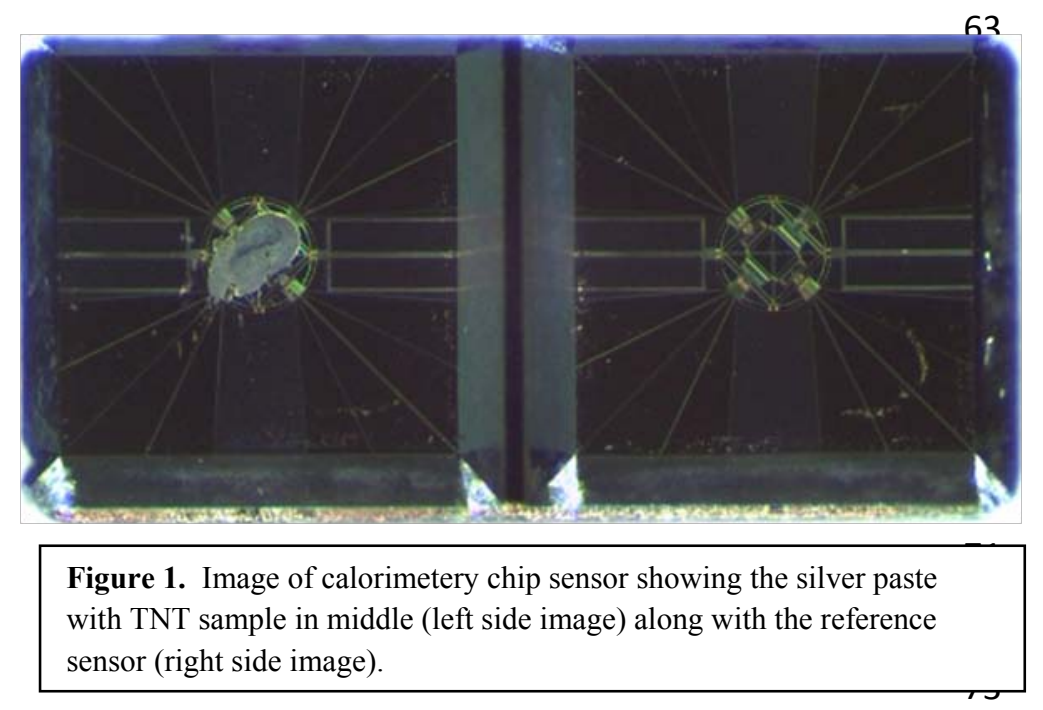

The silver paste was applied in a thin layer to the chip within the borders of the inner "diamond" of approximately $0.5 \mathrm{~mm} \times 0.5 \mathrm{~mm}$ area. This paste was applied using an animal hair provided with the instrument. Because the paste dries rapidly, the small crystal (approximately $0.3 \mathrm{~mm} \times 0.3 \mathrm{~mm}$ ) of TNT was placed onto the paste with the animal hair in a way that a slight

74 indent was created such that the liquid TNT when melted would not flow off of the sensor area. Figure 1

75 shows an image of the DSC chip with the silver paste and TNT sample along with the reference side of

76 the chip showing no sample on the sensor portion.

Analysis

The glass transition temperatures were determined from the fictive temperatures $T_{f}$ obtained on heating as a function of the cooling rate where the glass was formed, i.e., we use $T_{f}=T_{g}[1]$. At the high cooling rates used in the flash DSC, the overshoots in enthalpy can be large, which can lead to large errors in the values of $T_{f}$ unless care is taken in the analysis to match the liquid lines and the glass lines when comparing the responses at different cooling rates. In our methodology, we superpose all of the liquid and glass lines for all of the runs of a given sample for the different cooling rates. This technique also provides good liquid lines for samples cooled at low rates. The fictive temperatures are then determined using the same liquid and glass lines for all of the runs.

The values of $T_{f}$ were obtained using the Moynihan area matching method, which, through integration of equation 1, determines $T_{f}$ as the point of intersection of the glassy enthalpy with the extrapolated equilibrium enthalpy line: [1,3]

90

$$
\int_{T_{f}}^{T \gg T_{g}}\left(C_{p, l}-C_{p, g}\right) d T=\int_{T \ll T_{g}}^{T \gg T_{g}}\left(C_{p}-C_{p, g}\right) d T
$$


92 where $C_{p, l}$ is the liquid state heat capacity, $C_{p, g}$ is the glass state heat capacity, $C_{p}$ is the apparent heat

93 capacity as a function of temperature, $T$ is temperature, $T_{f}$ is fictive temperature and $T_{g}$ is the glass

94 transition temperature.

95 The glass temperature is a function of cooling rate and can be analyzed in the context of the Vogel[4],

96 Fulcher[5], Tammann[6] (VFT) equation as:

97

98

$$
q\left(T_{g}\right)=q_{0} \exp \left[\frac{-B}{T_{g}-T_{0}}\right]
$$

99

100

101

102

103

104

105

106

107

108

109

110

111

112

113

114

115

116

08

09

15

where $q\left(T_{g}\right)$ is the cooling rate associated with a given glass transition temperature $T_{g}$ (or fictive

temperature $\left.T_{f}\right), q_{0}, B$, and $T_{0}$ are fitting parameters, with the latter two having some physical significance and the other being thought of as a prefactor. In particular, for the VFT temperature dependence of the behavior, the $B$ parameter has a role similar to an activation energy $/ R$ (the universal gas constant), and the parameter $T_{0}$ is the VFT temperature where the relaxation time or viscosity would diverge to infinity, perhaps at an 'ideal' glass transition. Furthermore, from the VFT parameters, one can determine the activation energy at the glass temperature $E_{g}$ and the dynamic fragility $m$ of the material through the equations[7,8,9,10]:

$$
E_{g}=\frac{R B}{\left[1-T_{0} / T_{g}\right]^{2}}
$$

$$
m=\frac{B / T_{g}}{\ln (10)\left[1-T_{0} / T_{g}\right]^{2}}
$$

When the range of glass transition temperatures to be investigated is small enough (curvature on the plot of $\ln q$ vs. $1 / T_{f}$ is low), as the case here, the VFT equation can be replaced with an Arrhenius equation, in which case the data can be analyzed using the following equations $[11,12]$ :

$$
\begin{gathered}
q\left(T_{g}\right)=q_{0} \exp \left(\frac{-E}{R T_{g}}\right) \\
m=\frac{E_{g}}{\ln 10 R T_{g}}
\end{gathered}
$$




\section{RESULTS}

118 Glass Transition Temperature of TNT

119 Figure 2 shows the heat flow curves that

120 aresult upon heating the TNT material after

121 cooling at rates from $0.1 \mathrm{~K} / \mathrm{s}$ to $1000 \mathrm{~K} / \mathrm{s}$.

122 The first thing we remark upon is that for

123 cooling rates of $100 \mathrm{~K} / \mathrm{s}$ or above, we did not

124 get crystallization of the TNT upon reheating

125 at $600 \mathrm{~K} / \mathrm{s}$. Only the glass transition is seen

126 upon reheating of the TNT at $600 \mathrm{~K} / \mathrm{s}$. At

127 lower cooling rates two types of behavior are

128 seen. First, at $10 \mathrm{~K} / \mathrm{s}$ and $30 \mathrm{~K} / \mathrm{s}$ we see that

129 there is a glass transition event and, at

130 somewhat higher temperatures, one sees a

131 cold crystallization exotherm followed by a

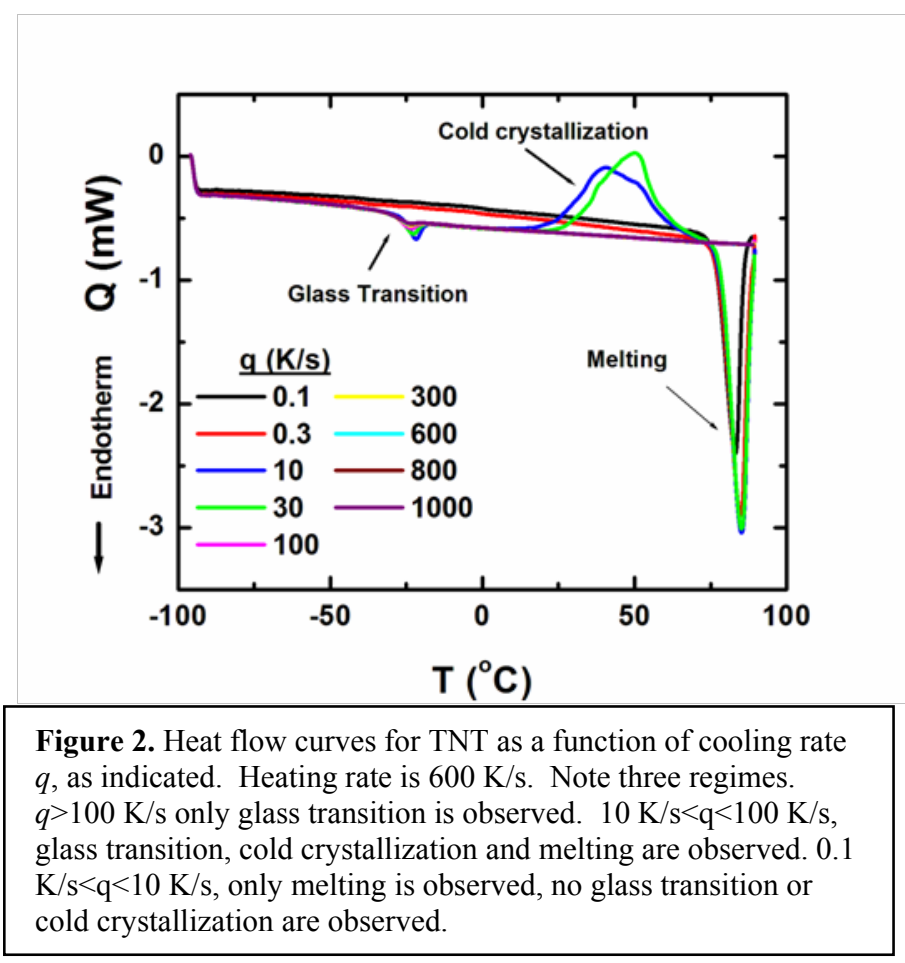

132 melting endotherm. At even lower cooling

133 rates $(\mathrm{q}=0.1$ and $0.3 \mathrm{~K} / \mathrm{s})$ one does not see the glass transition event and only melting is seen upon

134 reheating, indicating that full crystallization of the TNT occurred during cooling before the heating scan.

135 The cooling rate dependence of the fictive temperature for rates at and above $10 \mathrm{~K} / \mathrm{s}$ where a glass

136 transition event was observed is shown in Figure 3. Using the fact that $T_{g}=T_{f}$ we find that the $T_{g} \mathrm{~s}$ of TNT

137

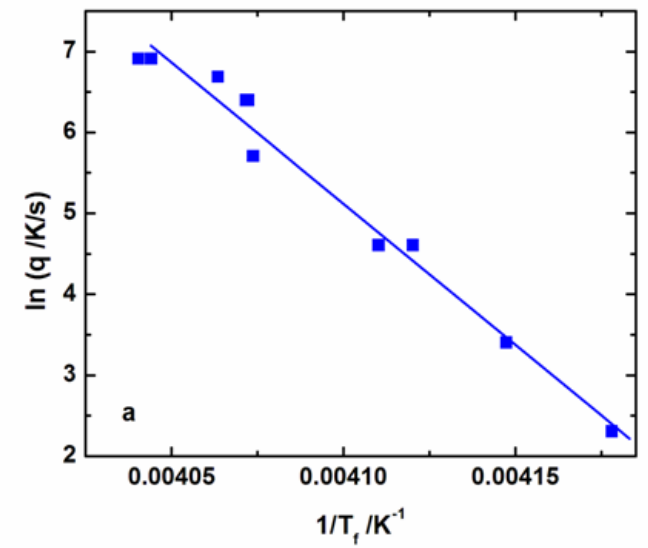

Figure 3. a) Plot of natural logarithm of the heating rate vs. the reciprocal of the fictive temperature for TNT. Line represents fit to equation 5. range from $239.3 \mathrm{~K}\left(-33.9^{\circ} \mathrm{C}\right)$ at $10 \mathrm{~K} / \mathrm{s}$ cooling

rate to $247.5 \mathrm{~K}\left(-25.7^{\circ} \mathrm{C}\right)$ at $1000 \mathrm{~K} / \mathrm{s}$ cooling rate. The activation energy is $290 \pm 16 \mathrm{~kJ} / \mathrm{mol}$ from the data in Figure 3 (Equation 5) and the dynamic fragility index is found to be $m=62 \pm 6$ (equation 6).

The value of $m \cong 62$, makes TNT a typical, small molecule, glass former [9]. It is much less fragile than, e.g., the extremely fragile polymer poly(ether imide)[13] where $m=214$, or the extremely fragile small molecule liquid decalin for which $m=145[11]$. 


\section{Cold Crystallization of TNT}

152 Though the main purpose of the present work was to establish the glass transition of TNT, we also

153 found that the cold crystallization response of this material upon heating from the glassy state depends

154 strongly on the cooling rate at which the glass is formed. Returning to Figure 1, we see three regimes of

155 behavior: q $>100 \mathrm{~K} / \mathrm{s}$ only glass transition is observed; $10 \mathrm{~K} / \mathrm{s}<\mathrm{q}<100 \mathrm{~K} / \mathrm{s}$, glass transition, cold

156 crystallization and melting are observed; $0.1 \mathrm{~K} / \mathrm{s}<\mathrm{q}<10 \mathrm{~K} / \mathrm{s}$, only melting is observed with no glass

157 transition or cold crystallization. While these results are not exhaustive, they are similar to results

158 reported for more complex glass formers, such as metallic glasses [14, 15, 16], and the behavior may also

159 be related to reports in simpler molecular glass formers of crystallization out of the glassy state that seems

160 faster than expected from VFT dynamics [17,18,19]. Clearly, the path to vitrification determines the

161 crystallization behavior as one might expect the formation of crystal nuclei or pre-nuclei to be different

162 depending on the detailed path of vitrification. Future work is planned using flash DSC to investigate in

163 more detail TNT and other rapidly crystallizing materials.

\section{DISCUSSION}

The present work presents, to our knowledge, the first direct measurement of the glass transition temperature of TNT and its variation with cooling rate. However, it is important to recognize that research on TNT in the late 1960s by May, Thorpe and Connick [22] estimated the $\mathrm{T}_{\mathrm{g}}$ of TNT to be -15.2 ${ }^{\circ} \mathrm{C}(258 \mathrm{~K})$ based on nominal cooling at $64 \mathrm{~K} / \mathrm{min}(1.07 \mathrm{~K} / \mathrm{s})$. Clearly, from the data presented here, this estimate is too high by at least $15-20 \mathrm{~K}$, but they did recognize that the material should vitrify when sufficiently supercooled to avoid crystallization. Importantly, they also reported that they saw evidence that the cold crystallization after vitrification led to a different heat of fusion from that expected for a conventionally crystallized sample, similar to the results reported above for the cooling rates at which cold crystallization was observed after devitrification (See Figure 1). Clearly, the work from May, Thorpe and Connick [22] and that of the present study show that TNT can be vitrified and that the details of the vitrification-devitrification path lead to changes in the thermal response of the material because the crystallization response is dependent on this path. How this thermal behavior might affect the behavior of TNT in use is probably not very important because near ambient storage and use temperatures for TNT are above the devitrification temperature and crystallization would be expected to occur. However, it is possible that other energetic materials having higher values of $T_{g}$ could exhibit a change in performance

182 (calorimetric response) changing during the heating cycle required for ignition or detonation of these 183 materials. Clearly, further investigation is warranted to examine these possibilities. 


\section{SUMMARY AND CONCLUSIONS}

Flash differential scanning calorimetry has been used to cool TNT at rates $q$ ranging from $0.01 \mathrm{~K} / \mathrm{s}$ to $1000 \mathrm{~K} / \mathrm{s}$, i.e., a range of five orders of magnitude. We find three regimes of behavior upon reheating are observed: $q>100 \mathrm{~K} / \mathrm{s}$ only a glass transition is observed; $10 \mathrm{~K} / \mathrm{s}<\mathrm{q}<100 \mathrm{~K} / \mathrm{s}$, glass transition, cold crystallization and melting are observed; $0.1 \mathrm{~K} / \mathrm{s}<\mathrm{q}<10 \mathrm{~K} / \mathrm{s}$, only melting is observed with no glass transition or cold crystallization. For the regimes where a glass transition is observed, we have been able to determine the activation energy of vitrification $E_{g}$ to be approximately $290 \pm 16 \mathrm{~kJ} / \mathrm{mole}$ and the dynamic fragility index is $m \cong 62 \pm 6$, similar to other small molecule organic liquids. For the cooling rate between $10 \mathrm{~K} / \mathrm{s}$ and $1000 \mathrm{~K} / \mathrm{s}$, the $T_{g}$ (i.e., $T_{f}$ ) varies from $239.3 \mathrm{~K}$ to $247.5 \mathrm{~K}$. These values are significantly lower than prior estimates in the early TNT literature. Future work is planned to investigate the influence of the vitrification path on cold crystallization above the $T_{g}$ as well as crystallization from the glassy state itself.

\section{ACKNOWLEDGEMENTS}

The authors are grateful to the Office of Naval Research under Project No. N00014-11-1-0424 and the John R. Bradford endowment at Texas Tech University, each for partial support of this work.

\section{REFERENCES}

1. P. Badrinarayanan, W. Zheng, Q. Li, S. L. Simon, "The glass transition temperature versus the fictive temperature," Journal of Non-Crystalline Solids, 353, 2603-2612 (2007).

2. S. Gao, Y. P. Koh, and S. L. Simon, "Calorimetric Glass Transition of Single Polystyrene Ultrathin Films," Macromolecules, 46, 562-570 (2013).

3. C.T. Moynihan, A.J. Easteal, M.A. DeBolt, J. Tucker, " Dependence of the Fictive Temperature of Glass on Cooling Rate," J. Am. Ceram. Soc., 59, 12-16 (1976).

4. H. Vogel, “Das Temperaaturabhängigkeitsgesetz der Viskosität Flüssigkeiten,” Phys. Z., 22, 645-646 (1921).

5. G. S. Fulcher, "Analysis of Recent Measurements of the Viscosity of Glasses," J. Am. Ceram. Soc., 8, 339-355 (1925).

6. G. Tammann, “Glasses as supercooled liquids,” J. Soc. Glass Technol., 9, 166-185 (1925). 
7. C. A. Angell, " Relaxation in liquids, polymers and plastic crystals--strong/fragile patterns and problems," J. Non-Cryst. Solids, 131, 13-31 (1991).

229

8. R. Bohmer, C. A. Angell, "Correlations of the nonexponentiality and state dependence of mechanical relaxations with bond connectivity in Ge-As-Se supercooled liquids," Phys. Rev. B, 4, 10091-10094 (1992).

9. Q. Qin, G. B. McKenna, "Correlation between dynamic fragility and glass transition temperature for different classes of glass forming liquids," J. Non-Cryst Solids, 352, 2977-2985 (2006).

10. V. Lubchenko, P. G. Wolynes, " Theory of structural glasses and supercooled liquids," Annu. Rev. glassforming liquids by differential scanning calorimetry: Kinetic versus thermodynamic fragilities," J. Chem. Phys., 117, 10184-10192 (2002).

12. A. K. Torres-Arellano and G.B. McKenna, "Extremely Fragile Glass-Formers? Calorimetric and

13. S.L. Simon, D.J. Plazek, J.W. Sobieski and E.T. McGregor, "Physical Aging of a Polyetherimide: Volume Recovery and Its Comparison to Creep and Enthalpy Measurements," J. Polym. Sci. B: Polym. Phys., 35, 929-936 (1997).

14. A. L. Greer, "Crystallization Kinetics of $\mathrm{Fe}_{80} \mathrm{~B}_{20}$ Glass," Acta Metall., 30, 171-192 (1982).

15. A. Inoue, "Stabilization of Metallic Supercooled Liquid and Bulk Amorphous Alloys," Acta Mater., 48, 279-306 (2000).

16. G. Kumar, D. Rector, R.D. Conner, J. Schroers, "Embrittlement of Zr-based Bulk Metallic Glasses," Acta Mater., 57, 3572-3583 (2009).

19. Y. Sun, H. Xi, S. Chen, M. D. Ediger and L.Yu, " Crystallization near Glass Transition: Transition from Diffusion-Controlled to Diffusionless Crystal Growth Studied with Seven Polymorphs," J. Phys. Chem. B, 112, 5594-5601 (2008).

20. Y. Sun, H. Xi, M. D. Ediger and L. Yu, "Diffusionless Crystal Growth from Glass Has Precursor in Equilibrium Liquid," J. Phys. Chem. B, 112, 661-664 (2008).

21. T. Wu, "Origin of Enhanced Crystal Growth Kinetics near $T_{g}$ Probed with Indomethacin Polymorphs," J. Phys. Chem. B, 110, 15694-15699 (2006). 
267 22. F.G.J. May, B.W. Thorpe and W. Connick, "A glass transition in trinitrotoluene," J.Crystal Growth, 268 5, 312-312 (1969). 


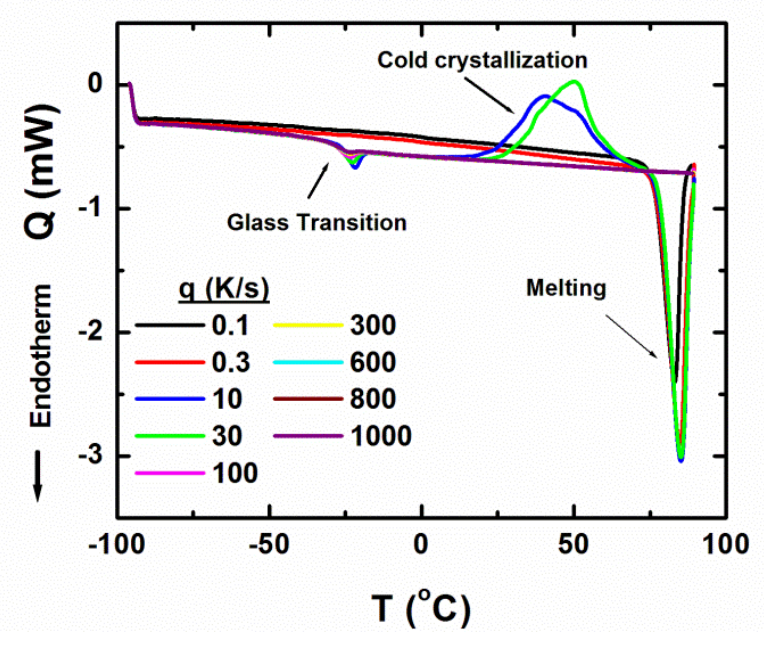

Caption. Flash DSC heating traces for TNT cooled at different rates. Three regimes of behavior evident: $q>100 \mathrm{~K} / \mathrm{s}$ only glass transition is observed. $10 \mathrm{~K} / \mathrm{s}<\mathrm{q}<100 \mathrm{~K} / \mathrm{s}$, glass transition, cold crystallization and melting are observed. $0.1 \mathrm{~K} / \mathrm{s}<\mathrm{q}<10 \mathrm{~K} / \mathrm{s}$, only melting is observed, no glass transition or cold crystallization are observed. 